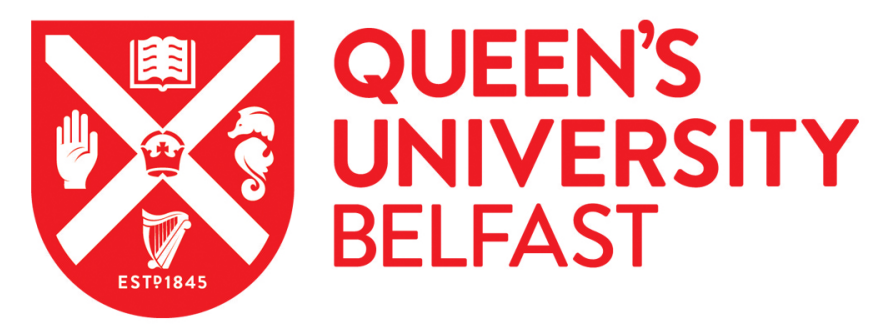

\title{
On Passive Intermodulation Test of Analog and Digital Systems
}

Kozlov, D., Shitvov, A., \& Schuchinsky, A. (2015). On Passive Intermodulation Test of Analog and Digital Systems. In 2015 Integrated Nonlinear Microwave and Millimetre-wave Circuits Workshop (INMMiC) Institute of Electrical and Electronics Engineers Inc.. https://doi.org/10.1109/INMMIC.2015.7330375

\section{Published in:}

2015 Integrated Nonlinear Microwave and Millimetre-wave Circuits Workshop (INMMiC)

\section{Document Version:}

Peer reviewed version

\section{Queen's University Belfast - Research Portal:}

Link to publication record in Queen's University Belfast Research Portal

\section{Publisher rights}

(c) 2015 IEEE. Personal use of this material is permitted. Permission from IEEE must be obtained for all other uses, in any current or future media, including reprinting/republishing this material for advertising or promotional purposes, creating new collective works, for resale or redistribution to servers or lists, or reuse of any copyrighted component of this work in other works.

\section{General rights}

Copyright for the publications made accessible via the Queen's University Belfast Research Portal is retained by the author(s) and / or other copyright owners and it is a condition of accessing these publications that users recognise and abide by the legal requirements associated with these rights.

Take down policy

The Research Portal is Queen's institutional repository that provides access to Queen's research output. Every effort has been made to ensure that content in the Research Portal does not infringe any person's rights, or applicable UK laws. If you discover content in the Research Portal that you believe breaches copyright or violates any law, please contact openaccess@qub.ac.uk. 


\title{
On Passive Intermodulation Test of Analog and Digital Systems
}

\author{
Dmitry Kozlov, Alexey Shitvov, and Alexander Schuchinsky \\ The Institute of Electronics, Communications and Information Technology (ECIT), \\ Queen's University Belfast, Belfast BT3 9DT, United Kingdom
}

\begin{abstract}
This paper presents initial results of evaluating suitability of the conventional two-tone $\mathrm{CW}$ passive intermodulation (PIM) test for characterization of modulated signal distortion by passive nonlinearities in base station antennas and RF front-end. A comprehensive analysis of analog and digitally modulated waveforms in the transmission lines with weak distributed nonlinearity has been performed using the harmonic balance analysis and X-parameters in Advanced Design System (ADS) simulator. The nonlinear distortion metrics used in the conventional two-tone CW PIM test have been compared with the respective spectral metrics applied to the modulated waveforms, such as adjacent channel power ratio (ACPR) and error vector magnitude (EVM). It is shown that the results of two-tone $\mathrm{CW}$ PIM tests are consistent with the metrics used for assessment of signal integrity of both analog and digitally modulated waveforms.
\end{abstract}

Index Terms - Communication nonlinearities, passive intermodulation, signal integrity, nonlinear distortion.

\section{INTRODUCTION}

In a wireless communications vocabulary, intermodulation is broadly defined as the generation of spectral components at the output of a nonlinear system incommensurate with the carrier frequencies of the input signal. It is typically a harmful phenomenon, since the spurious spectrum of the distorted transmit signals may intermingle with the regular signal at the transceiver front-end, causing strong interference in the adjacent channel and even rendering the link inoperable.

Although passive components produce significantly lower level of nonlinear signal distortion than active devices, they noticeably increase receiver noise floor, ultimately reducing the SNR and link capacity. The grave impact of passive nonlinearities is exacerbated by their obscure multi-physics that makes them extremely difficult to detect, locate, identify and mitigate by conventional means of PA linearization and testing.

The two-tone passive intermodulation test defined by the IEC62037 (1999) standard is routinely used for qualification of passive components of frequency division multiplexing systems. This test employs scalar measurement of a single combinatorial frequency in the Rx band resulting from mixing of two Tx carrier frequencies. The typical spec for the thirdorder PIM (PIM3) products is below $-112 \mathrm{dBm}$ at $2 \times 43 \mathrm{dBm}$ CW carriers. This basic PIM characterization does not give insight in the sources of passive nonlinearities, nor guarantees its applicability to modulated signals.

Although the shortcomings of two-tone CW PIM testing have long been recognised [1], there is a growing concern of suitability of the test for the emerging systems using broadband modulated signals, which often coexist with the legacy equipment [2]. On one hand, the signal waveforms become increasingly complicated and the signal integrity metrics are now required to capture the essential dynamics of the nonlinear distortions and effectively implement digital correction. On the other hand, complex modulated signals are more exposed to nonlinear distortion in passive devices. In this paper, we examine the utility of the two-tone CW PIM characterization of the $\mathrm{Rx}$ intermodulation distortion of analog and digital signals in nonlinear transmission line (NTL) with distributed passive nonlinearities using extended ACPR and EVM metrics.

\section{NuMERICAL MODEL AND METHODOLOGY}

The NTL is modelled as a cascade of the unit cells described by a low-pass equivalent circuit with capacitive nonlinearity of the third-order shown in Fig. 1. The unit cell model has been informed by our experimental studies of microstrip lines fabricated on the commercial RF laminates with dominant substrate (capacitive) nonlinearity and simulated with the aid of the X-parameters [3]. This agrees well with the assumption of weak nonlinearity, and discussion of more complex models and their physical bounds is presented in [4].

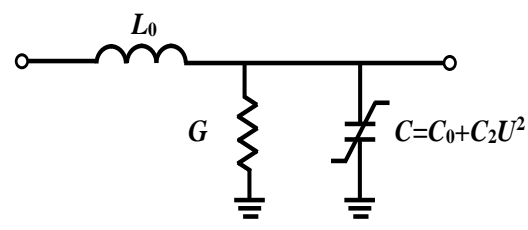

Fig. 1. The unit cell of a nonlinear transmission line.

The electrical length of the unit cell in Fig. 1, defined in terms of the phase shift $\theta$, is set equal to $45^{\circ}$, which ensures greater than $20 \mathrm{~dB}$ return loss in the frequency band from DC to $2 \mathrm{GHz}$.

Since we are interested in the effect of the distributed nonlinearity on the transmitted signals, we focus on the forward PIM products, i.e. the PIM signal measured at the TL output. We assume the Tx band spans 925 to $960 \mathrm{MHz}$ and Rx band is from 880 to $915 \mathrm{~Hz}$, which corresponds to the legacy EGSM900. The forward third order PIM (PIM3) products are generated by $2 \times 43 \mathrm{dBm} \mathrm{CW}$ carriers of frequencies $f_{1}=935 \mathrm{MHz}$ and $f_{2}=960 \mathrm{MHz}$. A $45^{\circ}$ phase shift at PIM3 frequency $2 f_{1}-f_{2}=910 \mathrm{MHz}$ is realized with the following circuit parameters of the $50 \Omega \mathrm{TL}: L_{0}=3.66 \mathrm{nH}, G=3 \cdot 10^{-5} \mathrm{~S}$, 
and $C_{0}=2.5 \mathrm{pF}$. The value of the nonlinear capacitance, $C_{2}=4.17 \cdot 10^{-6} \mathrm{pF} / \mathrm{V}^{2}$, has been chosen higher than suggested by the experimental data, cf. [3], in order to obtain discernible PIM3 level in a range of the NTL length values from $45^{\circ}$ to $720^{\circ}$ with different modulation schemes.

\section{ANALOG MODULATION}

At first, we consider analog amplitude modulated (AM) twotone test signal

$$
s(t)=\left[1+m_{a} \sin \left(2 \pi f_{m} t\right)\right] A\left[\cos \left(2 \pi f_{1} t\right)+\cos \left(2 \pi f_{2} t\right)\right]
$$

where $m_{a}$ is the modulation index. The modulating frequency $f_{m}=1 \mathrm{MHz}$ and carrier frequencies are $f_{1}=935 \mathrm{MHz}$ and $f_{2}=960 \mathrm{MHz}$. The spectrum of the output signal of a single $45^{\circ}$ NTL cell is shown in Fig. 2. The principal feature of the undistorted AM signal spectrum is the appearance of two sidebands about the carrier frequency, i.e. upper- and lowersideband lines, which are in phase and of equal magnitude depending on the modulation index and carrier amplitude. The results in Fig. 2 show the appearance of the spectral components around the frequencies corresponding to the oddorder PIM products. The spectrum near the frequency indicated by marker 'Mark2' corresponds to the lower-sideband PIM3 product, which falls within the Rx band. It is noteworthy that the PIM spectrum has a broader bandwidth. Also noticeable is the appearance of the additional spectral lines around each of the carriers due to the nonlinear mixing, which are much weaker than the input signal but symmetrical with respect to the carrier frequency. Note that the spectrum spread in frequency domain will compress the waveform in time domain.

To quantify the integrated spurious power associated with nonlinear signal distortion, we adopt the generalised ACPR metric for both two-tone $\mathrm{CW}$ and two-tone AM signals

$$
A C P R=P_{a c h} / P_{0}
$$

where $P_{a c h}$ is the total power in the Rx band and $P_{0}$ the total power across all Tx channels (the total power of the input signal). The latter is fixed at $46 \mathrm{dBm}$ throughout this paper, irrespective of the other parameters of the input signal (i.e., modulation index, number of carriers, etc.)

Figure 3 compares the simulated $A C P R$ as a function of the NTL length for the two-tone $\mathrm{CW}$ and AM signals. The two curves fully overlap, thus indicating that even though the PIM3 spectrum of the two-tone AM signal spreads across the Rx band, the total power down-converted to the RX band remains almost the same as for the two-tone $\mathrm{CW}$ test signal, given the same power of the input signal in both cases. This observation implies that the two-tone $\mathrm{CW}$ test actually gives accurate estimation of the distortion in the Rx band produced by the twotone AM signal, albeit it does not reveal the specifics of the spectral power density in the Rx band, nor it is capable of assessment of the nonlinear distortions in the Tx band.

As the second test, we use the phase-modulated (PM) signal of the following form:

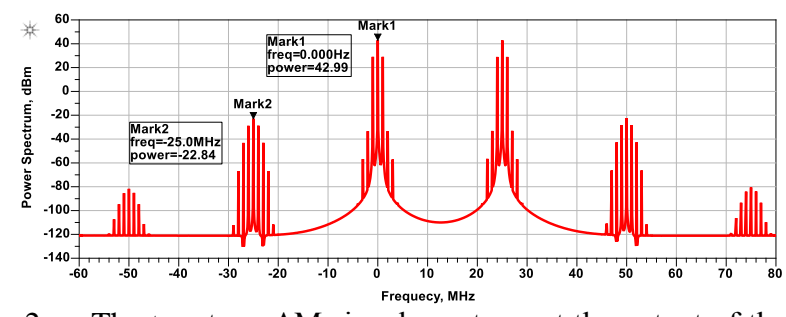

Fig. 2. The two-tone AM signal spectrum at the output of the $45^{\circ}$ NTL for $m_{a}=0.1$. Chart center (Marker 1) is at $935 \mathrm{MHz}$.

$$
s(t)=A\left[\cos \left(2 \pi f_{1} t+m_{p} \sin \left(2 \pi f_{m} t\right)\right)+\cos \left(2 \pi f_{2}+m_{p} \sin \left(2 \pi f_{m} t\right)\right)\right]
$$

The modulating and carrier frequencies, and the total input signal power are the same as above. A distinct characteristic of the spectrum of a PM signal is its infinite extent and out-phased sidebands. In the narrowband FM signal representation, which is valid for $m_{p}<0.5$, spectrum of the PM signal can be truncated to the two nearest sidebands and resembles an AM signal. However, the sidebands will not be in phase here. As the modulation index increases, the number of the significant sidebands will also increases.

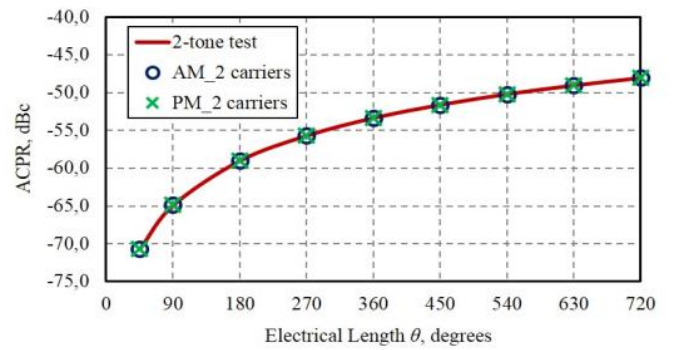

Fig. 3. Simulated ACPR for two-tone CW, AM and PM signals $\left(m_{a}=0.1\right)$.

Figure 4 shows the simulation results for the two-tone PM signal transmitted via a $45^{\circ}$ NTL section. The cusped power spectral density function in the Rx band reflects the spectra of the modulated carriers. It is also noteworthy that the carrier and PIM spectra appear asymmetrical, which suggests the AM-PM conversion due to the distributed nonlinearity. The AM spectrum can be deduced by de-embedding the phase modulation from the output signal. Despite showing rather different spectrum regrowth, the ACPR of the two-tone PM signal still coincides with that of the two-tone $\mathrm{CW}$ signal.

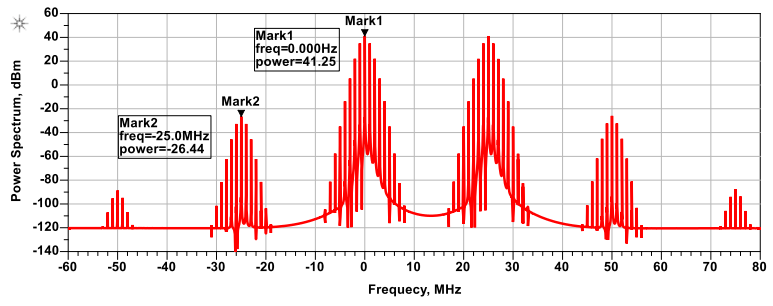

Fig. 4. The two-tone PM signal spectrum at the output of the $45^{\circ}$ NTL for $m_{p}=1$. Chart center (Marker 1) is at frequency $935 \mathrm{MHz}$.

This analysis has been further extended to the PIM products generated by the three-tone PM carriers of frequencies $f_{1}=935 \mathrm{MHz}, f_{2}=940 \mathrm{MHz}$ and $f_{3}=960 \mathrm{MHz}$, which 
corersponds to the Tx band of E-GSM $(925 \mathrm{MHz}-960 \mathrm{MHz})$. The output spectrum of a $45^{\circ}$ NTL is simulated for $m_{p}=1$ is shown in Fig. 5 and the corresponding ACPR are shown in Fig. 6. Note that the distortion spectrum is more evenly spread across the Rx band and beyond, which is reflected in the lower ACPR as compared with the three-tone $\mathrm{CW}$ signal test.

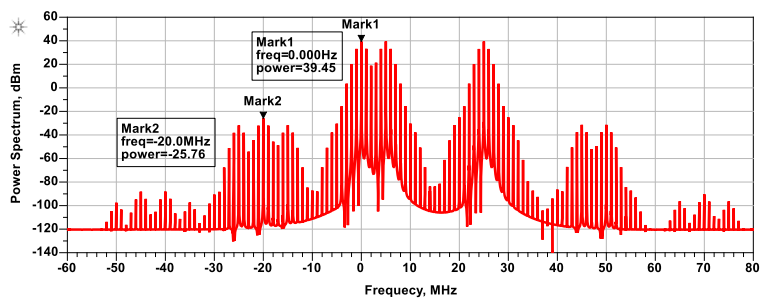

Fig. 5. The three-tone PM signal spectrum at the output of the $45^{\circ}$ NTL for $m_{p}=1$. Chart center (Marker 1) is at frequency $935 \mathrm{MHz}$.

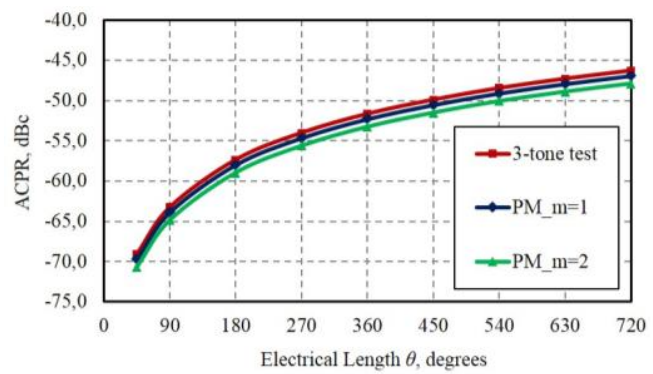

Fig. 6. Simulated ACPR for 3-tone CW \& PM signals $\left(m_{p}=1 \& 2\right)$

The latter observation implies that the multi-tone $\mathrm{CW}$ signal test provides a conservative evaluation of the distortion power in the Rx band and it is expected that the overestimate will increase with the number of carriers. When compared with the two-tone $\mathrm{CW}$ test, we note that the three-tone $\mathrm{CW}$ test produces slightly higher PIM level over a range of the line lengths, and this trend is likely to increase with the number of the carriers.

\section{Digital Modulation}

Distortion of digital signals is often characterized by EVM. In essence, the EVM represents a metric of the signal constellation deviations from the ideal one due to added signal impairments. If the received symbols cross the border of adjacent symbol decision region, this will cause error. As the result, higher order modulation schemes require higher signalto-noise ratio for the same probability of a symbol error [5].

A comparison of the ACPR values calculated for the twotone CW, two BPSK and two QPSK signals of equal total input power is shown in Fig. 7. It demonstrates that the two-tone CW test gives the same integrated spurious power in the receive band as that generated by mixing two digitally modulated signals.

EVM simulations using two-tone BPSK and QPSK signals of equal input power transmitted via the NTL of varying length are shown in Fig. 7. Since the forward PIM level grows with the NTL length, signal to noise ratio (SNR) is higher on the longer NTL at the given receive signal power. In the considered cases SNR varied from $40 \mathrm{~dB}$ (for $\theta=45^{\circ}$ ) to $18 \mathrm{~dB}$ (for $\theta=720^{\circ}$ ).

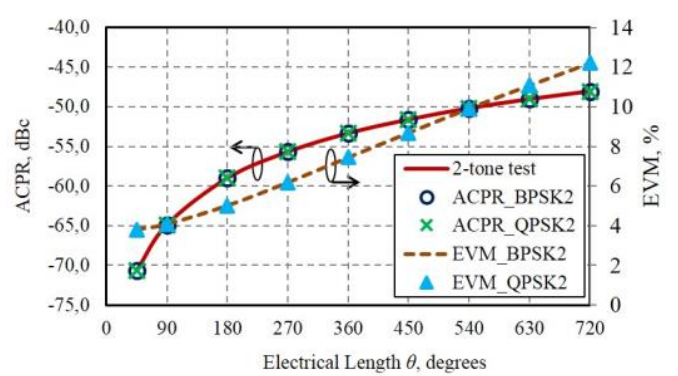

Fig. 7. Simulated ACPR and EVM for two-tone CW, two BPSK \& two QPSK signals transmitted via the NTL of the electrical length $\theta$.

\section{CONCLUSION}

The nonlinear mixing of analog and digitally modulated waveforms in a transmission line with passive distributed nonlinearity has been analyzed using different spectral and symbol metrics, i.e., ACPR and EVM. It is shown that the conventional two-tone CW PIM3 assessment is consistent with these metrics. The PIM products of multi-tone $\mathrm{CW}$ signal appear to have higher ACPR than those generated by the PM carriers with the same total input power. The presented results raise a question whether the discussed metrics are sufficient for assessment of the signal integrity of the modulated waveforms in the passive devices and if the two-tone CW PIM tests provide adequate conservative estimations of the PIM effect on the system performance. Influence of additive white noise in the Rx channel in comparison with IM distortions and filtering effect on EVM values will be further discussed in the presentation.

\section{ACKNOWLEDGEMENT}

This work has been performed in the framework of Marie Curie European Industrial Doctorate (EID) program ARTISAN jointly with Bell Labs Ireland, grant No. 316426. DK gratefully acknowledges support by the Marie Curie EID Fellowship.

\section{REFERENCES}

[1] D. Weinstein, "Passive Intermodulation Distortion in Connectors, Cable and Cable Assemblies", White Paper XP002330778, Amphenol Corp., February 2001.

[2] J.-J. DeLisle, "New Modulation Schemes Raise PIM," Microwaves \& RF, vol. 53, no. 3, p. 36, March 2014.

[3] D. Kozlov, A. Shitvov, and A. Schuchinsky, "Characterisation of Passive Intermodulation in Passive RF Devices with Xparameters", Loughborough. Antennas \& Propag. Conf., pp.6467, November 2014.

[4] J. Sombrin, "Non-analytic at the origin, behavioural models for active or passive non-linearity," Int. J. Microwave and Wireless Technologies, vol. 5, no. 2, pp. 133-140, 2013.

[5] R. A. Shafik, S. Rahman, and R. Islam, "On the Extended Relationships Among EVM, BER and SNR as Performance Metrics," 4th International Conference on Electrical and Computer Engineering ICECE, pp. 408-411, Dec. 2006. 